Helen H. J. Pothuizen · Joram Feldon • Benjamin K. Yee

\title{
Facilitated extinction of appetitive instrumental conditioning following excitotoxic lesions of the core or the medial shell subregion of the nucleus accumbens in rats
}

Received: 18 May 2005/ Accepted: 2 December 2005/Published online: 24 January 2006

(C) Springer-Verlag 2006

\begin{abstract}
The nucleus accumbens has been implicated in the control of goal-directed behaviour, including instrumental conditioning. Here, we evaluated the effect of $\mathrm{N}$-methyl-D-aspartate (NMDA)-induced excitotoxic lesions restricted to either the core or the medial shell subregions of the nucleus accumbens (NAC) on extinction in rats using a trial discrete fixed ratio-5 (FR-5) appetitive operant procedure. Neither core nor shell lesions of the NAC affected the acquisition of instrumental responding. Both lesions facilitated the cessation of responding when the instrumental act no longer yielded reinforcement. Our results suggest that both the NAC core and medial shell contribute to the control of extinction learning of appetitively motivated instrumental behaviour.
\end{abstract}

Keywords Behavioural switching - Extinction learning - Incentive motivation - Operant behaviour · Ventral striatum

\section{Introduction}

The nucleus accumbens (NAC) has been implicated in the control of goal-directed action (Mogenson et al. 1980; Kalivas and Nakamura 1999; Cardinal et al. 2002; Kelley 2004). In particular, it has been attributed a pivotal role in relating reward processes to the actionoutcome associations that underlie instrumental learning (Dickinson and Balleine 1994). Anatomically, the ascending mesolimbic dopaminergic inputs and descending glutamatergic inputs from limbic cortices converge onto the principle neurons of the NAC (e.g. Totterdell and Smith 1989; Meredith et al. 1990; Brog

H. H. J. Pothuizen · J. Feldon $(\bowtie) \cdot$ B. K. Yee Laboratory of Behavioural Neurobiology, Swiss Federal Institute of Technology Zurich, Schorenstrasse 16, 8603 Schwerzenbach, Switzerland E-mail: feldon@behav.biol.ethz.ch

Tel.: + 41-1-655-7448

Fax: + 41-1-655-7203 et al. 1993), which in turn project to the pallidum and via multiple synaptic connections influence executive areas including the premotor and prefrontal cortices (e.g. Deniau et al. 1994; Groenewegen et al. 1996). Dopaminergic activities in the NAC appear to be critically involved in the regulation and modulation of 'reward' processes mediating the incentive motivation of the animal to engage in an instrumental response (Horvitz 2000; Salamone and Correa 2002; Berridge and Robinson 2003; Kelley 2004), and instrumental performance is reduced by NAC dopamine depletion (e.g. Sokolowski and Salamone 1998).

The NAC's modulatory roles in instrumental conditioning have also been revealed by the use of selective outcome devaluation procedure (Corbit et al. 2001; also see de Borchgrave et al. 2002) and Pavlovian-instrumental transfer in rats with excitotoxic NAC lesions (Corbit et al. 2001; Hall et al. 2001). These reports suggest that the NAC plays a role in selecting or initiating actions based on the reward value of their consequences, and in mediating the guidance and activation by Pavlovian cues of goal-directed instrumental responding, although there is a discrepancy concerning the functional demarcation between the core and shell regions of the NAC. A more consistent negative finding, however, is the lack of an effect following NAC lesions on contingency degradation (Corbit et al. 2001; de Borchgrave et al. 2002).

Extinction represents another form of control over instrumental responding: one that is prompted by a specific change in the action-outcome relationship, namely, the complete removal of the primary reinforcers (Myers and Davis 2002; Bouton 2004), as opposed to contingency degradation by the additional delivery of noncontingent (free) rewards. Until now, the effects of discrete accumbens core or shell lesions on the extinction of simple instrumental conditioning have hardly been systemically investigated. In a runway experiment, Tai et al. (1991) reported that electrolytic accumbens lesions can lead to bidirectional changes in the rate of extinction depending on whether the animals were previously 
trained on a partial or a continuous reinforcement schedule. The extinction of conditional operant discrimination learning following large NAC lesions is, on the other hand, associated with various forms of response persistence (Annett et al. 1989; Reading et al. 1991).

Here, we examined the effects of discrete accumbens core or shell cytotoxic lesions on the extinction of a postoperatively acquired instrumental response. A trial-discrete lever-press procedure was adopted to ensure that all subjects had equivalent response-outcome experience during acquisition training prior to extinction. We evaluated the course of extinction learning from the initial reduction in response vigour to the eventual cessation of the operant behaviour.

\section{Methods}

\section{Subjects}

The subjects were naive male Wistar rats, weighing 280 $300 \mathrm{~g}$, obtained from our in-house specific pathogen-free breeding facilities. The animals were caged in groups of four and kept under a reversed 12/12 h light-dark cycle (lights on at $19.00 \mathrm{hrs}$ ). First, a cohort of 23 rats, divided into three groups (core lesions $=8$, medial shell lesions $=8$, sham controls $=7$ ) was tested. A second cohort of eight rats (core lesions $=4$, sham $=4$ ) was then added in a partial replication. All manipulations and procedures were carried out in the dark phase of the cycle, and had previously been approved by the Swiss Federal Veterinary Office. Every effort had been made to minimize the number of animals used.

\section{Surgery}

Bilateral lesions were made by intracerebral infusions of $N$-methyl-D-aspartate (NMDA, Sigma, St Louis, MN, USA) dissolved in $0.1 \mathrm{M}$ phosphate-buffered saline (PBS, $\mathrm{pH} 7.4$ ) at a concentration of $14 \mathrm{mg} / \mathrm{ml}$, under stereotaxic guidance as fully described by Jongen-Rêlo et al. (2003). The stereotaxic coordinates for the core lesions were: $0.10 \mu \mathrm{l}$ at $+1.4(\mathrm{AP}), \pm 2.0(\mathrm{ML}),-6.5$ (DV); and $0.075 \mu \mathrm{l}$ at \pm 2.4 (AP), \pm 1.8 (ML), -6.5 (DV). For the medial shell: $0.075 \mu \mathrm{l}$ at $+1.2(\mathrm{AP}), \pm 0.9$ (ML), -6.9 (DV); $0.075 \mu \mathrm{l}$ at $+1.2(\mathrm{AP}), \pm 0.9$ (ML), -6.6 (DV); and $0.025 \mu \mathrm{l}$ at $+1.2(\mathrm{AP}), \pm 0.9(\mathrm{ML}),-6.2$ (DV). Sham controls were infused with an equivalent volume of PBS at either the core or the medial shell coordinates. Behavioural testing commenced after 4 weeks of post-operative recovery.

\section{Apparatus}

Eight identical operant chambers (Coulbourn Instruments, Allentown, PA, USA); each set in a ventilated, sound- and light-attenuating cubicle, were used. Each chamber measured $30 \times 25 \times 21.5 \mathrm{~cm}$. Two light sources
$(1.8 \mathrm{~W})$ mounted on the manipulator panel wall, $19 \mathrm{~cm}$ above the grid floor, provided constant illumination throughout all test sessions. A magazine tray was positioned in the centre of the panel wall, equipped with a separate magazine light $(1.8 \mathrm{~W})$. An infrared beam detected magazine entry, and a pellet dispenser delivered 45-mg reward food pellets (Bioserv Frenchtown, NJ, USA) into the magazine. A retractable lever was positioned $3 \mathrm{~cm}$ away on either side of the magazine tray. Control of the chambers and collection of the data were accomplished by Graphic State Notation 1.013-00 software (Coulbourn) implemented on a personal computer running the Windows 98 operating system.

\section{Experimental procedures}

The two cohorts of subjects were tested approximately 8 weeks apart, and followed identical procedures as described in the following. Food deprivation was gradually introdived over the week before behavioural experimentation. The rats were eventually maintained with 1-h access to food per day. The weight of the animals was monitored weekly and not allowed to drop below $85 \%$ of their free feeding weight. Water was available ad libitum. Two days prior to the magazinetraining phase, the rats were familiarized with the reward pellets in their home cage.

Magazine training On day 1, 25 reward pellets were individually delivered into the magazine at a variable interval of $60 \pm 20 \mathrm{~s}$. Pellet delivery coincided with an 1-s illumination of the magazine light. The levers remained retracted throughout this session.

Lever press shaping On day 2, the animals received 25 discrete trials of lever-press training at an ITI of $30 \mathrm{~s}$. A trial began with the insertion of the left lever into the chamber, and one lever-press led immediately to the retraction of the lever and the delivery of a food pellet accompanied by an 1-s magazine light, i.e., a trial-discrete fixed ratio-1 (FR1) schedule. On day 3, the FR requirement was increased by steps of one after every five trials. All subjects acquired the final FR-5 response, and acquisition commenced the next day.

Acquisition Acquisition lasted for five consecutive days, with 12 discrete trials of FR-5 per day. A trial began with the presentation of the left lever. Following five lever-presses, the lever was retracted. A subsequent nose poke into the magazine tray was followed by the immediate delivery of four pellets accompanied by an 1-s magazine light. The next trial commenced following an inter-trial interval (ITI) of $60 \mathrm{~s}$. The entire operant act was subdivided into three segments: "start" refers to time of lever insertion to the first lever-press, "run" refers to the time from first to the fifth lever-press, and "goal" refers to the time from the fifth lever-press to magazine nose-poke. The subjects were allowed a maximum of $60 \mathrm{~s}$ to complete each segment of the operant 
act. Upon reaching the 60 -s limit, the trial would be aborted and resumed to the ITI; and all subsequent segment(s) of that trial would be assigned a criterion response latency of $60 \mathrm{~s}$.

Extinction Extinction began one day after the last acquisition day, and lasted for 6 days. The procedures were as described for acquisition training, except that completion of the operant act (which still resulted in retraction of the lever) was no longer followed by delivery of reward and illumination of the magazine light.

\section{Data analysis}

All three dependent variables (start, run, and goal times) were logarithmically transformed (base $e$ ) prior to parametric analysis of variance (ANOVA) with the between-subjects factor of treatment and the appropriate repeated measurements factors (days and four-trial blocks) using the statistical software SPSS (version 12) implemented on a PC running the Windows XP operating system. The acquisition and extinction phases were separately analysed, and so were the three variables corresponding to the three segments (i.e. start, run and goal) of the operant response. The final day of acquisition was included in the analysis of extinction. To allow a closer examination of the initial impact of extinction on responding, the three variables across the 12 trials (expressed in blocks of four trials) on the first day of extinction were further subjected to separate analysis.

All animals were tested across the 6 days of extinction test. Subsequently, a criterion of extinction was applied to determine the point of extinction. The operant response was considered extinguished when a subject failed to complete the entire operant act in three consecutive trials. In order to conform to, and to facilitate comparison with, our previous extinction studies of instrumental conditioning (e.g. Feldon and Weiner 1991), the response times of all subsequent trials and days of a subject that had extinguished were assigned to 60 s. Parallel analyses in which the criterion procedure was omitted essentially revealed identical patterns of statistical outcomes.

\section{Histological procedures}

At the end of the behavioural experiments the rats were deeply anaesthetized and perfused; their brain were extracted and processed for histological verification of the lesions' extent as described before (Jongen-Rêlo et al. 2003). Briefly, five adjacent series of coronal sections $(40 \mu \mathrm{m})$ were cut. One series was destined for cresyl violet staining to visualize cell bodies and another for immunohistochemical demonstration of the neuronalspecific nuclear protein (NeuN) as described in JongenRêlo and Feldon (2002) to allow a more accurate delineation of the lesions extent.

\section{Results}

Histological results

Reconstructions of the largest and the smallest core and medial shell lesions, based on subjects included in the behavioural analyses, are illustrated in Fig. 1a and b.

Core lesions The core lesions between the two cohorts were highly comparable, and no animal was excluded on histological grounds. All core lesions were characterized by extensive cell loss in the core and occasional shrinkage of this region and the anterior commissure. No damage was apparent in the shell. In three cases, the lateral ventricles were slightly enlarged in comparison with the other two groups, but no damage resulting from this ventricular enlargement was apparent in the surrounding brain areas. Limited damage to the dorsomedial aspect of the caudate putamen was observed in four animals, and such damage was restricted to a small area surrounding the needle track. A coronal section of a representative core lesion is depicted in Fig. 1c.

Medial shell lesions The lesions in two animals belonging to the medial shell lesion group were found to extend caudally into the ventral pallidum; they were therefore excluded from the behavioural analysis. In the remaining shell-lesioned subjects, the lesions were characterized by extensive gliosis and cell loss in the ventromedial part of the shell, sparing the ventrolateral aspect of the shell and the core. In two cases, limited cell damage in the adjoining olfactory tubercle was observed bilaterally. One subject also exhibited some unilateral damage to the lateral septum. A coronal section taken from a representative subject is depicted in Fig. 1d.

Sham controls No obvious cell damage to the NAC was evident in the sham controls in cohorts 1 and 2 (Fig. 1e). Mechanical damages to the cortical mantle were comparable with those seen in the medial shell and core groups. No sham controls were excluded from the final data analyses.

\section{Behavioural results}

The final analyses presented here combined data from cohorts 1 and 2 (Fig. 2). Before that, we had ascertained that no significant differences between cohorts emerged in analyses of all measures, of both acquisition and extinction phases, restricted to the sham- or the corelesioned subjects.

\section{Acquisition phase}

Start All groups showed a reduction of response time over days and across blocks (four trials within days), as evident by a significant effect of days $[F(4,104)=30.24$, $P<0.001]$ and of blocks $[F(2,52)=36.52, P<0.001]$. 


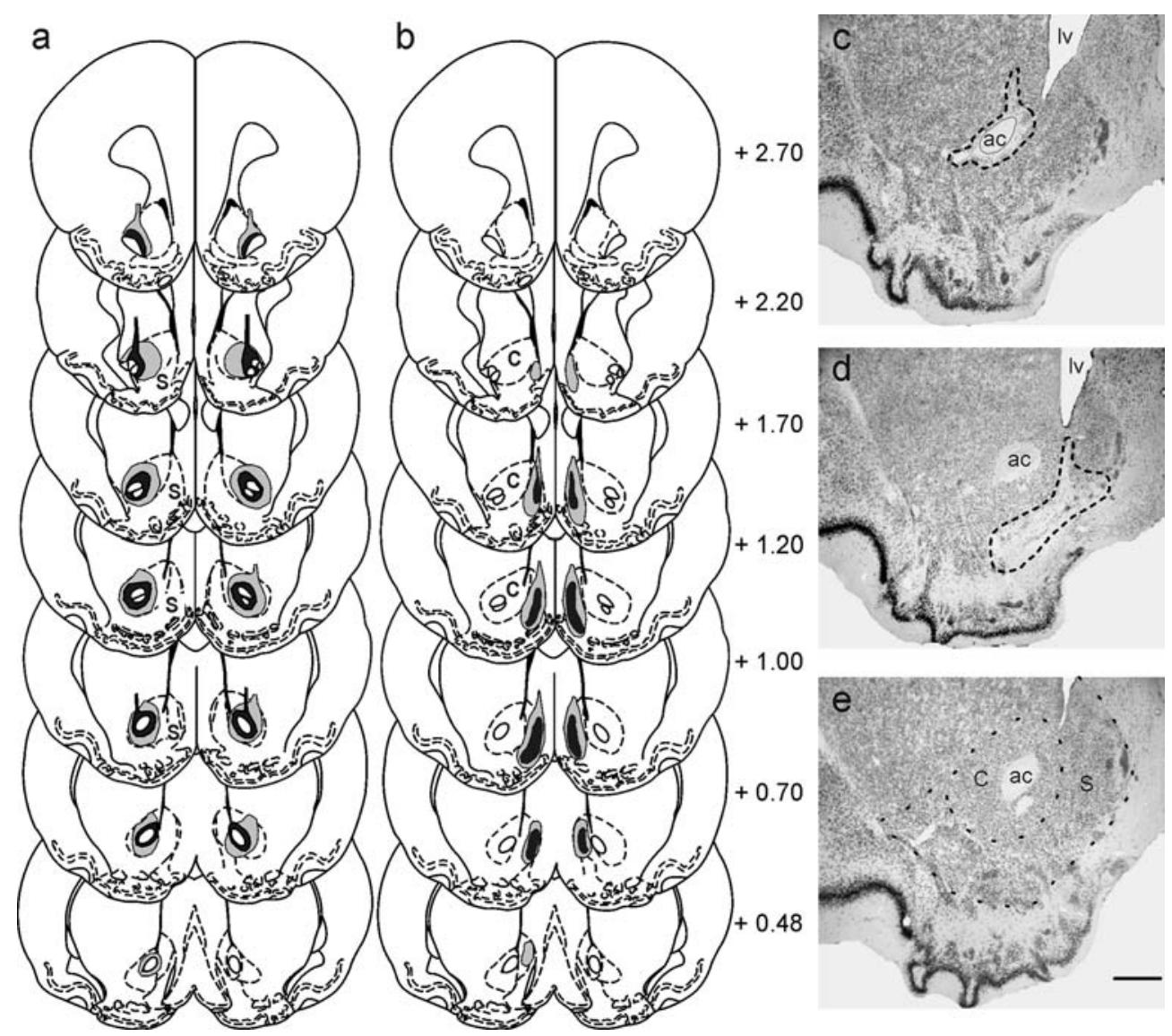

Fig. 1 Histological results. Reconstructions of the minimal (black) and maximal (black + grey areas) extents of the core and shell lesions included in the final analysis are illustrated in $\mathbf{a}$ and $\mathbf{b}$, respectively. These are mapped onto coronal plates at the specified anterior-posterior levels, adapted from Paxinos and Watson (1997) brain atlas. Photomicrographs of a coronal section through the NAC immunostained for the NeuN from a subject taken from the core lesioned group (c); medial shell lesions group (d) and a shamoperated control (e). Since the NeuN immunostaining selectively

Neither the main effect of lesion nor its interaction terms achieved statistical significance (all $F_{\mathrm{s}}<1$ ).

Run Acquisition of the operant response was evident by a reduction of response time in this segment over days $[F(4,104)=27.16, \quad P<0.001]$ and across blocks $[F(2,52)=14.52, \quad P<0.001]$. The interaction between days and blocks also achieved significance $[F(8,208)=3.57, P<0.001]$ because the reduction (within-days) across successive blocks gradually diminished over days. Again, neither the main effect of lesion $[F(2,26)=1.16, P=0.33]$ nor its interaction achieved statistical significance (minimal $P=0.26$ ).

Goal In this segment of the operant act, a significant reduction in response time across days was also evident $[F(4,104)=5.34, P<0.001]$, but it was relatively stable across blocks $(\mathrm{F}<1)$. Consistent with the analyses of the 'start' and 'run' time, neither the main effect of lesion labels neurons, the area of lesion can be readily discerned by the clear absence of immunoreactivity (as delineated by the dashed lines). The solid line in $\mathbf{c}$ outlines the boundary of the anterior commissure to facilitate its distinction from the area of lesion. The dotted line in e delineates the approximate anatomical boundary between the core and the shell subregion of the NAC. Scale bar in e applies to all photomicrographs and represents $500 \mu \mathrm{m}$. Abbreviations ac anterior commissure; $C$ core subregion; $l v$ lateral ventricle; $S$ shell subregion

$[F(2,26)=2.37, P=0.11]$ nor its interaction achieved statistical significance $\left(F_{\mathrm{s}}<1\right)$.

\section{Extinction phase}

Start The 'start' time increased as extinction progressed over days $[F(6,156)=201.0, P<0.001]$ and also across blocks $[F(2,52)=45.78, P<0.001]$. The interaction between days and blocks also attained statistical significance $[F(12,312)=12.66, P<0.001]$, because response time dropped over blocks on the last day of acquisition, but increased across successive blocks on all extinction days; and the latter effect of blocks was more pronounced in the initial days of extinction.

As shown in Fig. 2, the rate of extinction over days clearly differed among groups. Extinction was most rapid in the medial shell group followed by the core group, and was the slowest in the sham control group. This led 


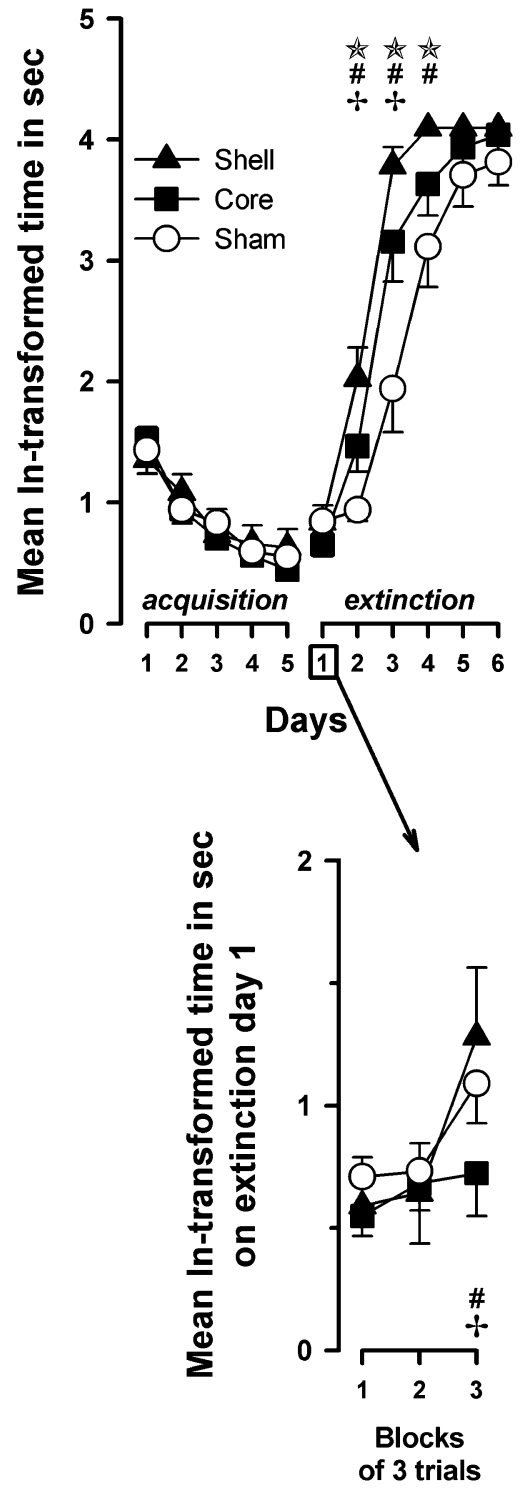

Fig. 2 Effects of selective medial shell and core lesions on the acquisition and extinction of a trial discrete FR-5 appetitive instrumental conditioning paradigm. The top row, from left to right, illustrates the mean response time [ln-transformed, max $=$ $\ln (60)=4.094]$ in the start, run, and goal segments of the operant act, respectively. The bottom row illustrates the mean ln-transformed response time across successive four-trial blocks on the first day of extinction at the corresponding segments. Error bars refer to \pm SEM of the corresponding mean values. Medial shell (filled triangle): $n=6$; Core (filled square): $n=12$; sham control (open

to the emergence of a significant main effect of lesion $[F(2,26)=4.98, P<0.05]$, and its interaction with days $[F(12,156)=3.66, P<0.001]$. The three-way interaction also achieved significance $[F(24,312)=2.55, P<0.001]$.

Additional restricted ANOVAs comparing sham controls directly with either of the shell or the core groups revealed that both lesions significantly facilitated

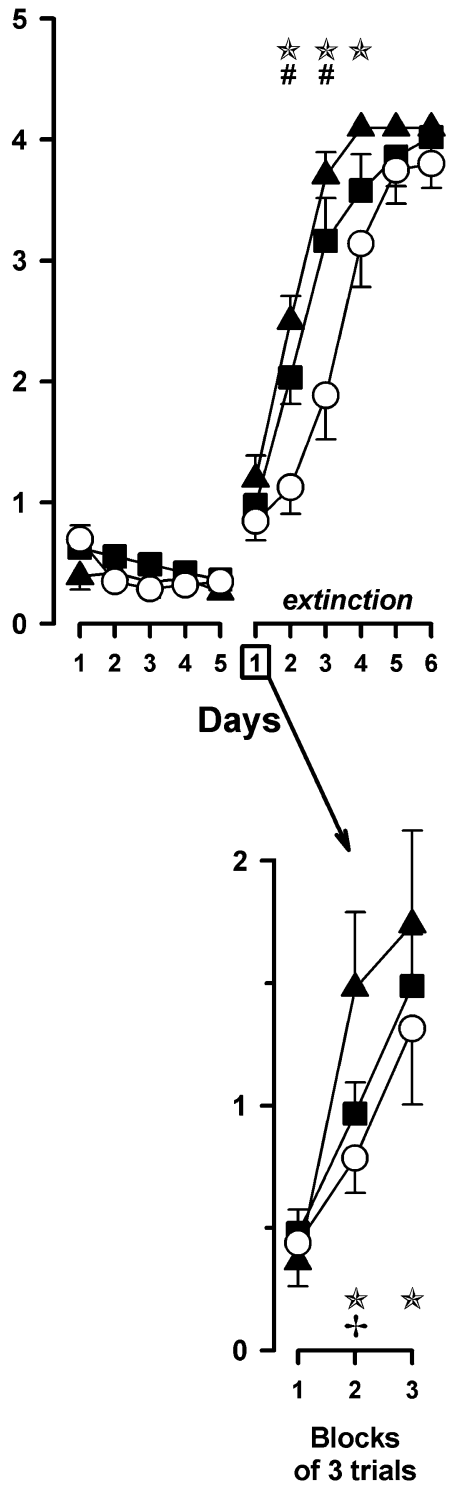

circle): $n=11$. Within each plot and on a given day (upper panel) or three-trial block (lower panel), the symbol t indicates a significant difference between shell and sham groups, the symbol \# indicates a significant difference between core and sham groups, and the symbol + indicates a significant difference between shell and core groups. These pair-wise comparisons were performed as described by Winer (1971), based on the pooled mean square error term associated with the lesion $\times$ days interaction in the corresponding ANOVA

extinction over days. In both analyses, the interaction term between lesion and days remained significant [shell versus sham: $F(6,90)=5.88, P<0.001$; core versus sham: $F(6,126)=3.75, P<0.005]$. The same interaction term was not statistically significant when the shell and core groups were directly compared $(\mathrm{F}<1)$, suggesting that their extinction curves did not differ from each other. 
However, post hoc pair-wise comparisons on successive extinction days indicated that the two lesioned groups differed significantly from each other on the second and third days of extinction $(P<0.05)$.

Run Similarly, the 'run' time increased as extinction progressed over days $[F(6,156)=189.4, P<0.001]$ and also across blocks within-days $[F(2,52)=70.75$, $P<0.001]$. The days $\times$ blocks interaction also attained statistical significance $[F(12,312)=10.79, P<0.001]$ for reasons as described previously. Importantly, both shell and core lesions also enhanced extinction in this segment of the operant response. This again yielded a significant interaction between lesion and days $[F(12,156)=3.35$, $P<0.001]$, accompanied by the main effect of lesion $[F(2,26)=4.85, P<0.05]$. The three-way interaction also achieved significance $[F(24,312)=3.03, P<0.001]$.

Separate statistical confirmations for the facilitating effect of shell and core lesions on extinction were again obtained by additional restricted ANOVAs as described previously. In both analyses, the interaction term between lesion and days was highly significant [shell versus sham: $F(6,90)=5.36, P<0.001$; core versus sham: $F(6,126)=3.25, P=0.005]$. The same interaction term did not attain statistical significance when the shell and core groups were directly compared $[F(6,96)=1.13$, $P=0.35$, suggesting that extinction curves of the two lesioned groups did not differ from each other. However, post hoc pair-wise comparisons on successive extinction days indicated that the two lesioned groups differed significantly from each other on the second and third days of extinction $(P<0.05)$.

Goal The pattern of results obtained in the goal section conformed closely to that seen in the start and run segments. A significant effect of days $[F(6,156)=168.6$, $P<0.001]$, of blocks $F(2,52)=152.2, P<0.001]$ and also their interaction $[F(12,312)=10.77, P<0.001]$ were obtained. The presence of a between-groups difference over the course of extinction was supported by the main effect of lesion $[F(2,26)=4.45, P<0.05]$, its interaction with days $[F(12,156)=3.32, P<0.001]$, and the three-way interaction $[F(24,312)=1.94, P<0.01]$. The effects of shell and core lesions on extinction were separately confirmed by additional restricted ANOVAs: each yielding a significant lesion $\times$ days interaction [shell versus sham: $F(6,90)=6.01, P<0.001$; core versus sham: $F(6,126)=3.31, P<0.005]$. The same interaction term was far from statistical significance when the shell and core groups were directly compared $(\mathrm{F}<1)$, suggesting that the two lesioned groups did not differ from each other.

\section{Extinction day 1}

The initial impact of extinction across the three blocks of the first extinction day is illustrated in the lower panels of Fig. 2 for the start, run, and goal segments.

A significant effect of blocks emerged in all segments [start: $F(2,52)=8.71, P<0.001 ;$ run: $F(2,52)=21.42$,
$P<0.001$; goal: $F(2,52)=29.49, P<0.001]$. A significant effect of lesions was only apparent in the run segment in the form of a significant lesion $\times$ blocks interaction $[F(4,52)=2.98, P<0.05]$. To examine the separate contributions of shell and core lesions to the emergence of this significant effect, restricted ANOVAs confining to two out of the three groups were conducted. These indicated that this effect stemmed primarily from a contrast between shell and core lesions [shell versus core: lesion $\times$ blocks interaction $=F(2,32)=5.16, P=0.01$ ], while neither the shell nor the core groups differed significantly from sham controls. Post hoc comparisons in the last block of the run segment suggested that when shell-lesioned animals were responding significantly slower than sham control, core-lesioned animals were responding faster (see lower panel of Fig. 2). The latter effect was also apparent in the start segment. Other significant pair-wise differences between shell and core groups were also noted in both the start and goal segments, despite the lack of an overall lesion $\times$ blocks interaction in these segments.

\section{Discussion}

The present study showed that discrete cell body lesions confined to either the core or medial shell subregion of the NAC facilitated the extinction of a post-operatively acquired appetitive instrumental response. This effect was essentially demonstrated against the background of a lack of an effect in the acquisition phase; and therefore, it cannot be attributed to deficiency or over-training in the acquisition phase. The results suggest that the effect following core lesions was as clear as that seen after medial shell lesions in statistical terms, although graphically the shell group consistently outpaced the core group in its rate of extinction. This was most clearly seen on the second and third days of extinction in the start and run segments of the operant response (see Fig. 2). Upon closer examination, performance on the first extinction day provided further evidence that the shell and core groups responded differently, and that the impact of non-reward appeared weaker in the corelesioned animals compared with sham controls, at least in the start and run segments. Thus, while selective lesions of NAC shell or core exert similar effects on extinction of an appetitive operant response across days, they can be distinguished in the immediate reaction to extinction. This hint of a weak resistance to extinction following core (but not shell) lesions may explain why the core group exhibited a marginally weaker facilitation effect across subsequent extinction days when compared with the shell group.

In this respect, it is worth pointing out that the trend towards a more robust effect following shell lesions cannot be accounted for simply by lesion size. Indeed, if the core and shell subregions of NAC were functionally homogeneous (at least in the present behavioural test), an opposite trend would be expected, because the core 
lesions, if anything, involved a wider area of cell loss than the medial shell lesions. Hence, although the present study suggested that both shell and core subregions of the NAC can both facilitate extinction, the possibility that (i) cell bodies within the shell subregion may be more tightly involved, and (ii) damage to core region is in addition associated with an effect on extinction that is opposite in direction, cannot be completely excluded.

Corbit et al. (2001) reported that core lesions disrupted the selective outcome devaluation effect in a twochoice procedure in which devaluation not only led to suppression of responding to the "devalued" lever, but also to the "non-devalued" lever. Given that the test was conducted in extinction, the lesion's effect on the nondevalued lever resembled our finding of enhanced extinction by core lesions. What appears inconsistent is our observation that shell lesions also enhanced extinction, whereas no such effect was apparent in Corbit et al.'s study. In addition, these authors reported an acquisition deficit following core lesions (also see Kelley et al. 1997; Hernandez et al. 2005), which is also in contrast to our present finding, but this difference is likely to stem from their use of a random-ratio (RR) schedule with a decreasing probability for each lever press to earn a reinforcement. In contrast, we employed an FR5 schedule here. Given the sensitivity of core lesions to non-reward as reflected here, it is not inconsistent that an acquisition deficit may emerge under certain reinforcement schedules, in which the animals may be confronted with frequent omissions of expected rewards (e.g. in a progressive RR schedule, see Corbit et al. 2001).

Core lesions have been reported to reduce tolerance to delayed reinforcement (Cardinal et al. 2001). This may also predict that the impact of reinforcement omission during extinction was stronger in the core-lesioned animals, and thereby leading to the observed facilitation of extinction. In a direct comparison between selective shell and core lesions, we reported that intolerance to delayed reward was only seen after core but not shell lesions (Pothuizen et al. 2005). Again, this may hint at a psychological difference underlying the effect on extinction observed here: while the facilitation of extinction seen following core lesions may be related to increased sensitivity to some forms of non-reward, the effect on extinction induced by selective shell lesions may stem from enhanced behavioural switching (see Weiner 1990, 2003).

The present finding however appears to contradict Tai et al.'s (1991) report that NAC lesions led to increased resistance to extinction following continuous reinforcement. Our trial-discrete FR-5 procedure ensured that reinforcement was earned in every completed trial, and the animals learned to reliably complete the five lever-presses before initiating the nose-poke response. Hence, our acquisition regime closely parallels the continuous reinforcement procedure that Tai et al. (1991) adopted in their straight alleyway paradigm. Apart from the difference in the employed apparatus, it should also be emphasized that the lesions studied by Tai et al. (1991) were made by electrolytic methods, and might have therefore revealed additional effects attributable to interruption of fibres en passage. However, it should be emphasized that Tai et al. (1991) also observed reduced resistenence to extinction in animals with NAC lesion when the instrumental response was acquired under a partial reinforcement schedule.

In another study, Annett et al. (1989) showed that large cell lesions of the NAC encompassing both shell and core did not lead to excessive perseveration during reversal of a T-maze discrimination task, but retarded the slowing of responding when all rewards were removed altogether. Apart from different lesion sizes, the fact that extinction was assessed following acquisition of a conditional discrimination task in which not only avoidance and approach responses were learned (see also Reading et al. 1991), but also an acquisition impairment was shown, precludes any straightforward interpretation of extinction learning in the experiments reported by Annett et al. (1989).

In sum, there is considerable evidence to suggest that both shell and core lesions can interfere with instrumental learning, including its extinction, but the direction of the effect observed also appears to depend on the reinforcement schedule involved. Here, the combination of highly selective lesions, the use of a simple (rather than discriminative) instrumental paradigm, and the lack of any clear disruption in the acquisition phase represents a unique contribution to the limited literature on NAC lesions and extinction. In addition, the present design allows the evaluation of extinction learning from the initial reduction in the vigour of the acquired instrumental response to its eventual cessation.

Myers and Davis (2002) have emphasized the distinction between 'within-session' extinction that perhaps reflects the short-term effect of reinforcement omission, and the long-term consolidation of extinction. To allow a closer evaluation of extinction in the short term, we have also examined the immediate impact of reinforcer omission by focussing on the 12 trials on the first extinction day. On this day, evidence for any substantial lesion effect was weak. First, a significant effect was only revealed in the 'run' segment, but not in the other two segments of the operant act. Second, this significant effect in the 'run' segment was primarily attributed to a difference between the two selective lesions, with neither lesions differing significantly from controls. In comparison, the effects on extinction by both selective lesions were most clearly expressed over days, and might therefore be more closely related to the consolidation and/or retention of extinction learning. Nonetheless, the immediate impact of reward omission following NAC lesions certainly deserves further investigation, for example by varying the amount of initial acquisition training, or initiating extinction midway within a session.

The relevance of the present study is not limited to the neural bases of motivational guidance of 
goal-directed behaviour, including the extinction of instrumental conditioning. It also bears implications on several accounts of NAC function in terms of behavioural switching and behavioural flexibility (Robbins et al. 1990; Weiner 1990, 2003; Weiner and Feldon 1997). Accordingly, facilitated extinction may be considered as a form of enhanced behavioural switching away from the control by the response $\rightarrow$ outcome contingency prevalent during acquisition, which can be viewed as a form of reduced proactive interference. Our present data agree with Weiner's $(1990,2003)$ prediction that selective shell lesions can result in enhanced behavioural switching, but deviate from her assumption that behavioural inflexibility should follow selective core damage, even though there is evidence that shell, but not core, lesions facilitated extinction in the early phase of extinction learning. In conclusion, further studies on extinction of learned behaviour are warranted to fully explore the functional significance of NAC (including the relative contributions of its subregions) as the link between motivation and action (Mogenson et al. 1980) as well as that between de-motivation and in-action.

Acknowledgements The authors thank the animal facility team for the excellent care of the animals, Mr. Peter Schmid for his technical assistance, Ms. Liz Weber for preparing the histology, and Mrs. Misa Kuper-Yamanaka for her editorial assistance. The research was supported by the Swiss Federal Institute of Technology Zurich and the Swiss National Science Foundation, with additional support from the National Centre of Competence in Research (NCCR): Neural Plasticity and Repair, Swiss National Science Foundation.

\section{References}

Annett LE, McGregor A, Robbins TW (1989) The effects of ibotenic acid lesions of the nucleus accumbens on spatial learning and extinction in the rat. Behav Brain Res 31:231-242

Berridge KC, Robinson TE (2003) Parsing reward. TINS 26:507513

Bouton ME (2004) Context and behavioral processes in extinction. Learn Mem 11:485-494

Brog JS, Salyapongse A, Deutch AY, Zahm DS (1993) The pattern of afferent innervation of the core and the shell in the 'accumbens' part of the ventral stiatum: immunohistochemical detection of retrogradely transported fluoro-gold. J Comp Neurol 338:255-278

Cardinal RN, Parkinson JA, Hall J, Everitt BJ (2002) Emotion and motvation. The role of the amygdala, ventral striatum, and prefrontal cortex. Neurosci Biobehav Rev 26:321-352

Cardinal RN, Pennicott DR, Sugathapala CL, Robbins TW, Everitt BJ (2001) Impulsive choice induced in rats by lesions of the nucleus accumbens core. Science 292:2499-2501

Corbit LH, Muir JL, Balleine BW (2001) The role of the nucleus accumbens in instrumental conditioning: evidence of a functional dissociation between accumbens core and shell. J Neurosci 21:3251-3260

de Borchgrave R, Rawlins JNP, Dickinson A, Balleine B (2002) Effects of cytotoxic nucleus accumbens lesions on instrumental conditioning in rats. Exp Brain Res 144:50-68

Deniau JM, Menetrey A, Thierry AM (1994) Indirect nucleus accumbens input to the prefrontal cortex via the substantia nigra pars reticulata: a combined anatomical and electrophysiological study in the rat. Neuroscience 61:533-545
Dickinson A, Balleine B (1994) Motivational control of goal-directed action. Anim Learn Behav 22:1-18

Feldon J, Weiner I. (1991) Effects of haloperidol on the multitrial partial reinforcement extinction effect (PREE): evidence for neuroleptic drug action on nonreinforcement but not on reinforcement. Psychopharmacology (Berl) 105:407-414

Groenewegen HJ, Wright CI, Beijer AVJ (1996) The nucleus accumbens: gateway for limbic structures to reach the motor system? Prog Brain Res 107:485-511

Hall J, Parkinson JA, Connor TM, Dickinson A, Everitt BJ (2001) Involvement of the central nucleus of the amygdala and nucleus accumbens core in mediating Pavlovian influences on instrumental behaviour. Eur J Neurosci 13:1984-1992

Hernandez PJ, Andrzejewski ME, Sadeghian K, Panksepp JB, Kelley AE (2005) AMPA/kainate, NMDA, and dopamine D1 receptor function in the nucleus accumbens core: A contextlimited role in the encoding and consolidation of instrumental memory. Learn Mem 12:285-295

Horvitz JC (2000) Mesolimbocortical and nigrostriatal dopamine responses to salient non-reward events. Neuroscience 96:651656

Jongen-Rêlo AL, Feldon J (2002) Specific neuronal marker: A new tool for histological evaluation of excitotoxic lesions. Physiol Behav 76:449-456

Jongen-Rêlo AL, Kaufmann S, Feldon J (2003) A differential involvement of the shell and core subterritories of the nucleus accumbens of rats in memory processes. Behav Neurosci 117:150-168

Kalivas PW, Nakamura M (1999) Neural systems for behavioral activation and reward. Curr Opin Neurobiol 9:223-227

Kelley AE, Smith-Roe SL, Holahan MR (1997) Response-reinforcement learning is dependent on $N$-methyl-D-aspartate receptor activation in the nucleus accumbens core. Proc Natl Acad Sci 94:12174-12179

Kelley AE (2004) Ventral striatal control of appetitive motivation: role in ingestive behavior and reward-related learning. Neurosci Biobehav Rev 27:765-776

Meredith GE, Wouterlood FG, Pattiselanno A (1990) Hippocampal fibers make synaptic contacts with glutamate decarboxylase-immunoreactive neurons in the rat nucleus accumbens. Brain Res 513:329-334

Mogenson GJ, Jones DL, Yim CY (1980) From motivation to action: functional interface between the limbic system and the motor system. Prog Neurobiol 14:69-97

Myers KM, Davis M (2002) Behavioral and neural analysis of extinction. Neuron 36:567-584

Paxinos G, Watson C (1997) The rat brain in stereotaxic coordinates. Academic Press, San Diego CA

Pothuizen HHJ, Jongen-Relo A, Feldon J, Yee BK (2005) Double dissociation of the effects of selective nucleus accumbens core and shell lesions on impulsive-choice behaviour and salience learning in rats. Eur J Neurosci 22:2605-2616

Reading PJ, Dunnett SB, Robbins TW (1991) Dissociable roles of the ventral, medial and lateral striatum on the acquisition and performance of a complex visual stimulus-response habit. Behav Brain Res 45:147-161

Robbins TW, Giardini V, Jones GH, Reading P, Sahakian BJ (1990) Effects of dopamine depletion from the caudate-putamen and nucleus accumbens septi on the acquisition and performance of a conditional discrimination task. Behav Brain Res $38: 243-261$

Salamone JD, Correa M (2002) Motivational views of reinforcement: implications for understanding the behavioural functions of nucleus accumbens dopamine. Behav Brain Res 137:3-25

Sokolowski JD, Salamone JD (1998) The role of accumbens dopamine in lever pressing and response allocation: Effects of 6OHDA injected into core and dorsomedial shell. Pharmacol Biochem Behav 59:557-566

Tai C-T, Clark AJM, Feldon J, Rawlins JNP (1991) Electrolytic lesions of the nucleus accumbens in rats which abolish the PREE enhance the locomotor response to amphetamine. Exp Brain Res 86:333-340 
Totterdell S, Smith AD (1989) Convergence of hippocampal and dopaminergic input onto identified neurons in the nucleus accumbens of the rat. J Chem Neuroanat 2:285-298

Weiner I (1990) Neural substrates of latent inhibition: the switching model. Psychol Bull 108:442-461

Weiner I (2003) The "two-headed" latent inhibition model of schizophrenia: modeling positive and negative symptoms and their treatment. Psychopharmacology 169:257-297
Weiner I, Feldon J (1997) The switching model of latent inhibition: an update of neural substrates. Behav Brain Res 88:11-25

Winer BJ (1971) Statistical principles in experimental design. McGraw-Hill, New York 